\title{
DYNAMICAL STRUCTURE OF VISCOUS ACCRETION DISKS WITH SHOCKS
}

\author{
Santabrata Das ${ }^{1}$, Peter A. Becker ${ }^{2,5}$, and Truong Le ${ }^{3,4}$ \\ ${ }^{1}$ Korea Astronomy and Space Science Institute, 61-1, Hwaam Dong, Yuseong-Gu, Daejeon 305 348, South Korea; sbdas@kasi.re.kr \\ ${ }^{2}$ Department of Computational and Data Sciences, George Mason University, Fairfax, VA 22030-4444, USA; pbecker@gmu.edu \\ ${ }^{3}$ Space Telescope Science Institute, 3700 San Martin Drive, Baltimore, MD 21218, USA; tle@stsci.edu \\ ${ }^{4}$ Computer Sciences Corporation, 45154 Underwood Lane, Sterling, VA 20166-9514, USA \\ Received 2008 May 22; accepted 2009 June 29; published 2009 August 14
}

\begin{abstract}
We develop and discuss global accretion solutions for viscous advection-dominated accretion flow (ADAF) disks containing centrifugally supported isothermal shock waves. The fact that such shocks can exist at all in ADAF disks is a new result. Interestingly, we find that isothermal shocks can form even when the level of viscous dissipation is relatively high. In order to better understand this phenomenon, we explore all possible combinations of the fundamental flow parameters, such as specific energy, specific angular momentum, and viscosity, to obtain the complete family of global solutions. This procedure allows us to identify the region of the parameter space where isothermal shocks can exist in viscous ADAF disks. The allowed region is maximized in the inviscid case, and it shrinks as the level of viscous dissipation increases. Adopting the canonical value $\gamma=1.5$ for the ratio of specific heats, we find that the shock region disappears completely when the Shakura-Sunyaev viscosity parameter $\alpha$ exceeds the critical value $\sim 0.27$. This establishes for the first time that steady ADAF disks containing shocks can exist even for relatively high levels of viscous dissipation. If an isothermal shock is present in the disk, it would have important implications for the acceleration of energetic particles that can escape to power the relativistic jets commonly observed around underfed, radio-loud black holes. In two specific applications, we confirm that the kinetic luminosity lost from the disk at the isothermal shock location is sufficient to power the observed relativistic outflows in M87 and Sgr A*.
\end{abstract}

Key words: accretion, accretion disks - black hole physics - galaxies: jets - hydrodynamics

\section{INTRODUCTION}

The accretion of matter onto compact objects is the fundamental mechanism powering a variety of high-energy astrophysical sources, such as low-mass X-ray binaries, massive black holes, and active galactic nuclei. Shakura \& Sunyaev (1973) and Novikov \& Thorne (1973) laid the foundation for our understanding of these sources by developing the first physical models for geometrically thin accretion disks. These early models did not treat the pressure and advection terms in the conservation equations correctly, and furthermore no attempt was made to satisfy the inner boundary conditions at the event horizon. Instead, the disk was simply terminated at the marginally stable orbit. The inner regions of these disks were shown to be viscously and thermally unstable by Lightman \& Eardley (1974), and the model was subsequently improved by Paczyński \& BisnovatyiKogan (1981) and Muchotrzeb \& Paczyński (1982) who incorporated advection and pressure effects into their models. Following a similar approach, the general global solution for accretion in the hydrodynamic limit, including advection, viscosity, and thermal effects, was obtained by Chakrabarti (1990, 1996).

\subsection{Advection-Dominated Accretion}

The advection-dominated accretion flow (ADAF) model remains a central paradigm in contemporary studies of blackhole accretion as a possible explanation for the dynamical structure of sources with significantly sub-Eddington accretion rates (see Yuan 2007 for a review). The gas in the inner regions of such disks is quite tenuous, and therefore the plasma radiates very

\footnotetext{
5 Also at: Department of Physics and Astronomy, George Mason University, Fairfax, VA 22030-4444, USA
}

inefficiently, leading to high temperatures. At large radii, the hot ADAF inner region is expected to merge with a cool, geometrically thin Shakura \& Sunyaev outer region characterized by a Keplerian angular momentum profile. Since any global accretion solution onto a black hole must cross the event horizon supersonically in order to satisfy the fundamental boundary conditions imposed by general relativity, ADAF accretion disks are necessarily transonic. Narayan et al. (1997) studied the behavior of transonic ADAF disks subject to the Shakura-Sunyaev viscosity prescription. The associated asymptotic boundary conditions governing the dynamics close to the event horizon, and the related physical restrictions on the viscosity prescription, were examined by Becker \& Subramanian (2005) and Becker \& Le (2003). Interest in the ADAF model remains strong, and it has been the focus of many recent studies. For example, Narayan \& McClintock (2008) have analyzed the properties of ADAF disks close to the event horizon; Takahashi (2007) has explored the influence of causal viscosity prescriptions on the structure of ADAF disks; Ma et al. (2007) have probed the role of magnetically induced torques in ADAF disks; and Yuan et al. (2008) have examined the properties of a simplified global model that utilizes an approximate form for the radial momentum equation.

\subsection{Critical Structure of Transonic Disks}

Due to the supersonic nature of the inflow at the event horizon, any physically achievable flow configuration must contain at least one critical point, where the flow transitions from subsonic to supersonic. However, for a given set of incident flow parameters (e.g., specific energy, specific angular momentum, and viscosity), the flow may possess multiple critical points (Chakrabarti 1989a, 1989b, 1990, 1996; Chakrabarti \& Das 2004; Le \& Becker 2005). This suggests the possible existence 
of global flow solutions with a standing shock wave located between two critical points. In their early study of viscous, transonic ADAF disks, Narayan et al. (1997) focused on global solutions that pass through a single critical point, and consequently they did not obtain any shocked disk solutions. The problem of the structure of ADAF disks governed by the Shakura-Sunyaev viscosity prescription was recently reexamined by Becker et al. (2008), who demonstrated that isothermal shocks can exist in such disks even in the presence of substantial viscosity.

In the present paper, we extend the approach taken by Becker et al. (2008) by performing a complete analysis of the structure of viscous ADAF disks containing either single or multiple critical points. Solutions with multiple critical points may include a standing shock, if it is possible to satisfy the shock jump conditions at some radius $r_{*}$ between the outer critical point (at radius $r=r_{c}^{\text {out }}$ ) and the inner critical point (at radius $\left.r=r_{c}^{\text {in }}\right)$. Shocks occur when the accretion flow is impeded due to the presence of a "centrifugal barrier" situated close to the horizon. Although such barriers can exist even in viscous disks, they begin to dissipate as the level of viscosity increases because of the increasing efficiency of the outward diffusion of angular momentum. Hence disks with large viscosity parameters are not expected to possess shocks, and our results confirm this. In the post-shock region, the flow accelerates toward the black hole, eventually passing through the inner critical point and crossing the horizon supersonically.

\subsection{Shock Waves in Viscous Disks}

The question of the stability of viscous accretion flows containing standing shock waves is currently not resolved. However, an important new development in the field was provided by Nagakura \& Yamada (2008), who used both linear stability analysis and general relativistic hydrodynamical simulations to investigate the stability properties of shock waves in inviscid, advection-dominated accretion flows around Schwarzschild black holes. When subjected to strong perturbations around the initial condition, the authors find that the dynamical configuration is stable, in the sense that the shock continues to exist, although the shock radius may oscillate if the perturbations are non-axisymmetric. Similar results were obtained by Okuda et al. (2007). Although these authors do not consider the effect of viscosity in their work, their conclusions nonetheless support the general idea that shocks may be able to exist in a stable configuration in relativistic disks. Consequently their findings provide a sound theoretical basis for the study of the structure of shocked, viscous disks developed in the present paper. When a shocked solution is dynamically possible, we argue based on the second law of thermodynamics that a shock will form since shocked-disk solutions possess higher entropy than smooth solutions (Becker \& Kazanas 2001).

The study of shock waves in accretion flows around compact objects has been undertaken by a variety of authors using either analytical methods (Fukue 1987; Chakrabarti 1989a, 1989b, 1990, 1996; Abramowicz \& Chakrabarti 1990; Nobuta \& Hanawa 1994; Yang \& Kafatos 1995; Yuan et al. 1996; Caditz \& Tsuruta 1998; Kovalenko \& Lukin 1999; Das et al. 2001; Das \& Chakrabarti 2004; Le \& Becker 2004, 2005, 2007; Yu et al. 2006; Becker et al. 2008) or numerical simulation (Hawley et al. 1984a, 1984b; Chakrabarti \& Molteni 1993; Molteni, Sponholz, \& Chakrabarti 1996; Ryu et al. 1997; Chakrabarti et al. 2004). The physical configuration of the shock depends on the detailed microphysics of the dissipation mechanism op- erating at the discontinuity. The three types of shocks most frequently studied are (1) Rankine-Hugoniot shocks, (2) isentropic shocks, and (3) isothermal shocks (Chakrabarti 1990; Abramowicz \& Chakrabarti 1990). Rankine-Hugoniot shocks possess the largest post-shock temperatures because the energy flux is strictly conserved as the gas crosses the shock. Hence shocks of this kind are radiatively very inefficient. In an isentropic shock, the entropy generated via dissipation is immediately radiated away, and consequently such shocks display smaller temperature increases than the Rankine-Hugoniot variety. In an isothermal shock, the temperature does not increase at all because all of the dissipated energy and entropy escape from the flow at the shock location. Hence isothermal shocks represent the most efficient mechanism for removing energy from the flow.

Le \& Becker $(2004,2005,2007)$ demonstrated that the energy removed from the flow at the isothermal shock location can be understood physically as a consequence of the firstorder Fermi acceleration of relativistic particles in the disk, combined with the diffusion and escape of the accelerated particles through the upper and lower surfaces of the disk. This approach facilitates the development of a completely self-consistent, coupled model that simultaneously describes the dynamical structure of the disk/shock system and also the energy spectrum of the relativistic particles escaping from the disk to form the observed jet outflows.

The properties of isothermal shock waves in accretion disks have been studied previously by Abramowicz \& Chakrabarti (1990), Lu \& Yuan (1998), Fukumura \& Tsuruta (2004), and Das et al. (2003). However, the studies cited above all focused on inviscid flow models, which probably do not adequately describe real accretion disks. In this paper, we obtain for the first time detailed dynamical solutions for the structure of ADAF disks containing isothermal shocks. The remainder of the paper is organized as follows. In Section 2 we discuss the ADAF model assumptions, the governing equations, and the critical conditions for the structure of ADAF disks. We discuss the isothermal shock jump conditions and examine global accretion solutions in Section 3, and we explore the properties of the standing shock and classify the parameter space for shocked accretion solutions in Section 4. Our dynamical formalism is used to model the development of the relativistic outflows in M87 and Sgr A* in Section 5, and concluding remarks are presented in Section 6.

\section{GOVERNING EQUATIONS}

We consider a thin, axisymmetric, viscous advectiondominated accretion disk structure. The disk is tenuous because it accretes matter at well below the Eddington rate, and therefore energy losses due to radiative processes are inefficient. General relativistic effects are taken into account in an approximate manner by utilizing the pseudo-Newtonian potential introduced by Paczyński \& Wiita (1980). The self-gravity of the disk is neglected. Under these assumptions, there are two conserved quantities in viscous ADAF disks, namely the mass transport rate

$$
\dot{M}=4 \pi r H \rho v,
$$

and the angular momentum transport rate

$$
\dot{J}=\dot{M} r^{2} \Omega-\mathcal{G}
$$

where $r$ is the radial coordinate, $H$ denotes the disk halfthickness, $\rho$ is the mass density, $v$ is the (positive) inflow 
velocity, $\Omega$ is the angular velocity, and $\mathcal{G}$ denotes the torque. The energy transport rate is given by

$$
\dot{E}=-\mathcal{G} \Omega+\dot{M}\left(\frac{1}{2} v^{2}+\frac{1}{2} v_{\phi}^{2}+\frac{P+U}{\rho}+\Phi\right),
$$

where $v_{\phi}=r \Omega$ denotes the azimuthal velocity, $P=(\gamma-1) U$ is the pressure, $U$ is the internal energy density, and $\Phi$ represents the pseudo-Newtonian potential, defined by (Paczyński \& Wiita 1980)

$$
\Phi(r) \equiv-\frac{G M}{r-r_{\mathrm{s}}},
$$

for a black hole with mass $M$ and Schwarzschild radius $r_{\mathrm{s}}=$ $2 G M / c^{2}$. This potential successfully reproduces many aspects of the spacetime geometry around a nonrotating black hole. We assume that the adiabatic index, $\gamma$, maintains a constant value throughout the flow, and the transport rates $\dot{M}, \dot{J}$, and $\dot{E}$ are all defined to be positive for inflow. Under our assumptions, $\dot{M}$ and $\dot{J}$ are conserved throughout the disk, and therefore they represent the rates at which mass and angular momentum enter the black hole, respectively. In general, the energy transport rate $\dot{E}$ is also conserved, except at the location of an isothermal shock if one is present in the flow.

The torque $\mathcal{G}$ is associated with the gradient of the angular velocity $\Omega$ through the standard relation (e.g., Frank et al. 2002)

$$
\mathcal{G}(r)=-4 \pi r^{3} H \rho v \frac{d \Omega}{d r},
$$

where $v$ is the kinematic viscosity. In the present work, we adopt the Shakura \& Sunyaev (1973) viscosity prescription,

$$
v(r)=\frac{\alpha a^{2}}{\Omega_{\mathrm{K}}},
$$

where $\alpha$ is a constant, $\Omega_{\mathrm{K}}$ denotes the Keplerian angular velocity, defined by

$$
\Omega_{\mathrm{K}}^{2}(r) \equiv \frac{G M}{r\left(r-r_{\mathrm{s}}\right)^{2}}=\frac{1}{r} \frac{d \Phi}{d r},
$$

and $a$ denotes the isothermal sound speed, given by

$$
a^{2}(r)=\frac{P}{\rho} .
$$

Combining Equations (1), (2), (5), and (6), we find that the gradient of the angular velocity can be written as

$$
\frac{d \Omega}{d r}=-\frac{v \Omega_{\mathrm{K}}\left(\ell-\ell_{0}\right)}{\alpha r^{2} a^{2}},
$$

where $\ell \equiv r^{2} \Omega$ denotes the angular momentum per unit mass for the accreting gas at radius $r$, and $\ell_{0} \equiv \dot{J} / \dot{M}$ is the angular momentum transport rate per unit mass. Since the torque $\mathcal{G}$ vanishes at the event horizon (Becker \& Le 2003), it follows from Equation (2) that $\ell_{0}$ is also equal to the specific angular momentum of the material entering the black hole. The radial derivative of $\ell$ is given by

$$
\frac{d \ell}{d r}=2 r \Omega+r^{2} \frac{d \Omega}{d r},
$$

which can be combined with Equation (9) to obtain the differential equation

$$
\frac{d \ell}{d r}=\frac{2 \ell}{r}-\frac{v \ell_{\mathrm{K}}\left(\ell-\ell_{0}\right)}{\alpha a^{2} r^{2}},
$$

where the Keplerian specific angular momentum $\ell_{\mathrm{K}}$ is defined by

$$
\ell_{\mathrm{K}}(r) \equiv r^{2} \Omega_{\mathrm{K}}(r)=\frac{r^{3 / 2} \sqrt{G M}}{r-r_{\mathrm{s}}} .
$$

The disk half-thickness $H$ is estimated using the standard vertical hydrostatic relation

$$
H(r)=\frac{a r^{2}}{\ell_{\mathrm{K}}} .
$$

In a steady state, the comoving radial acceleration rate in the frame of the accreting gas can be written as

$$
\frac{D v}{D t} \equiv-v \frac{d v}{d r}=\frac{1}{\rho} \frac{d P}{d r}+\frac{\ell_{\mathrm{K}}^{2}-\ell^{2}}{r^{3}} .
$$

By eliminating the torque $\mathcal{G}$ between Equations (2) and (3) and utilizing Equation (8), we find that the energy transport rate per unit mass is given by

$$
\epsilon \equiv \frac{\dot{E}}{\dot{M}}=\frac{1}{2} v^{2}-\frac{1}{2} \frac{\ell^{2}}{r^{2}}+\frac{\ell_{0} \ell}{r^{2}}+\frac{\gamma}{\gamma-1} a^{2}+\Phi .
$$

Since we are working within the ADAF framework, $\epsilon$ will remain constant unless a shock is formed in the disk. In this scenario, the variation of the internal energy density $U$ is regulated by the adiabatic compression of the gas and the viscous dissipation in the disk. The comoving rate of change of $U$ can therefore be written in the frame of the gas as (e.g., Becker \& Le 2003)

$$
\frac{D U}{D t} \equiv-v \frac{d U}{d r}=-\gamma \frac{U}{\rho} v \frac{d \rho}{d r}+\frac{\rho v}{r^{2}}\left(\frac{d \ell}{d r}-\frac{2 \ell}{r}\right)^{2} .
$$

We can obtain the dynamical differential equation governing the spatial variation of the inflow velocity $v$ by manipulating the conservation equations as follows. First we combine Equations (1), (13), and (8) to express the pressure as

$$
P=\frac{\dot{M} a \ell_{\mathrm{K}}}{4 \pi r^{3} v} .
$$

Using this relation to substitute for the pressure in Equation (14) for the radial acceleration, we find that the derivative of the sound speed $a$ is given by

$$
\frac{d a}{d r}=\left(\frac{a^{2}}{v^{2}}-1\right) \frac{v}{a} \frac{d v}{d r}-\frac{a}{\ell_{\mathrm{K}}} \frac{d \ell_{\mathrm{K}}}{d r}+\frac{3 a}{r}+\frac{\ell^{2}-\ell_{\mathrm{K}}^{2}}{a r^{3}} .
$$

Next we differentiate Equation (15) and combine the result with Equation (11) to obtain

$$
\frac{d v}{d r}=\frac{\ell^{2}-\ell_{\mathrm{K}}^{2}}{v r^{3}}-\frac{2 \gamma}{\gamma-1} \frac{a}{v} \frac{d a}{d r}-\frac{\ell_{\mathrm{K}}\left(\ell-\ell_{0}\right)^{2}}{\alpha a^{2} r^{4}} .
$$

By using Equation (18) to substitute for $d a / d r$ in Equation (19), we obtain the differential dynamical equation (e.g., Narayan et al. 1997)

$$
\begin{aligned}
\left(\frac{v^{2}}{a^{2}}-\frac{2 \gamma}{\gamma+1}\right) & \frac{1}{v} \frac{d v}{d r}=\frac{2 \gamma}{\gamma+1}\left(\frac{3}{r}-\frac{1}{\ell_{\mathrm{K}}} \frac{d \ell_{\mathrm{K}}}{d r}\right) \\
+ & \left(\frac{\gamma-1}{\gamma+1}\right) \frac{v \ell_{\mathrm{K}}\left(\ell-\ell_{0}\right)^{2}}{\alpha a^{4} r^{4}}+\frac{\ell^{2}-\ell_{\mathrm{K}}^{2}}{a^{2} r^{3}} .
\end{aligned}
$$

In order to obtain the distributions of the flow variables $v, a$, and $\ell$, we must solve numerically the governing Equations (11), (15), and (20). This requires consideration of the critical structure of the flow as discussed below. 


\subsection{Critical Conditions}

A black hole will swallow matter either from its binary companion or from the winds of the surrounding stars. At the outer edge of the disk, the accreting matter has negligible radial velocity, although it ultimately crosses the event horizon with a local velocity equal to the speed of light. Hence accretion flows around black holes must be transonic. The locations where the flow transitions from subsonic to supersonic are referred to as critical points. Depending on the parameters describing the properties of the gas at a large distance from the black hole, the flow may possess multiple critical points. When this occurs, it is interesting to investigate the possible existence of isothermal standing shock waves located between two of the critical points. The location of the shock, if one exists, is determined by applying the shock jump conditions. We first study the critical structure of the transonic flow by rewriting Equation (20) in the equivalent form

$$
\frac{d v}{d r}=\frac{N}{D}
$$

where the numerator and denominator functions $N$ and $D$ are defined by

$$
\begin{aligned}
N \equiv & \frac{2 \gamma v}{\gamma+1}\left(\frac{3}{2 r}+\frac{1}{r-r_{\mathrm{S}}}\right) \\
& \quad+\left(\frac{\gamma-1}{\gamma+1}\right) \frac{v^{2} \ell_{\mathrm{K}}\left(\ell-\ell_{0}\right)^{2}}{\alpha a^{4} r^{4}}+\frac{v\left(\ell^{2}-\ell_{\mathrm{K}}^{2}\right)}{a^{2} r^{3}},
\end{aligned}
$$

and

$$
D \equiv \frac{v^{2}}{a^{2}}-\frac{2 \gamma}{\gamma+1} .
$$

In order to obtain well-behaved global solutions, the numerator and denominator functions must vanish at exactly the same location in the flow. This condition represents the mathematical definition of a critical point. Setting $N=D=0$, we obtain the critical conditions

$$
\begin{aligned}
& \frac{2 \gamma v_{c}}{\gamma+1}\left(\frac{3}{2 r_{c}}+\frac{1}{r_{c}-r_{\mathrm{S}}}\right)+\left(\frac{\gamma-1}{\gamma+1}\right)\left[\frac{v_{c}^{2} \ell_{\mathrm{K}}\left(\ell_{c}-\ell_{0}\right)^{2}}{\alpha a_{c}^{4} r_{c}^{4}}\right] \\
& \quad+\frac{v_{c}\left(\ell_{c}^{2}-\ell_{\mathrm{K}}^{2}\right)}{a_{c}^{2} r_{c}^{3}}=0,
\end{aligned}
$$

and

$$
\frac{v_{c}^{2}}{a_{c}^{2}}-\frac{2 \gamma}{\gamma+1}=0
$$

where $r_{c}, v_{c}, a_{c}$, and $\ell_{c}$ denote the values of the radius, the velocity, the sound speed, and the specific angular momentum at the critical point, respectively. Any accretion solution must pass smoothly through the critical point, which is guaranteed if the critical conditions are satisfied. In this work, we assume approximate equipartition between the gas and magnetic pressures following Narayan et al. (1997), and accordingly we set $\gamma=1.5$ in the subsequent analysis.

The topological nature of a critical point is determined by the value of the velocity gradient $d v / d r$ at that point (e.g., Das 2007; Becker \& Le 2003). In general, we obtain two possible values for $d v / d r$, with one corresponding to accretion and the other to an outflow (wind). Any physically acceptable solution must pass through a saddle type or X-type critical point which is obtained when both of the derivatives are real and of opposite signs. If a shock forms, then the accretion flow must also pass through another saddle-type critical point in the post-shock region. When both of the values of $d v / d r$ are complex, the critical point is referred to as O-type. This type of critical point is unphysical and therefore no acceptable flow solution can pass through such a point.

\subsection{Inner Boundary Conditions}

Solutions for the flow variables $v(r), \ell(r)$, and $a(r)$ are obtained by numerically integrating Equations (11) and (20), supplemented by the algebraic relation given by Equation (15). Becker \& Le (2003) determined that in order to ensure the stability of the calculation, the integration must proceed in the outward direction, starting from a point close to the event horizon. This requires the availability of asymptotic boundary conditions that can be used to establish the starting values for the physical variables. Based on the fundamental general relativistic principle that the torque must vanish at the horizon (Weinberg 1972), Becker \& Le (2003) derived the explicit asymptotic behaviors of $v(r), \ell(r)$, and the "entropy function," $K(r)$, for material subject to the Shakura-Sunyaev viscosity prescription, where

$$
K(r) \equiv \frac{r v a^{\frac{\gamma+1}{\gamma-1}}}{\Omega_{\mathrm{K}}} .
$$

The physical significance of $K$ can be understood by combining Equations (1), (13), (8), and (26) to show that

$$
K^{\gamma-1} \propto \frac{P}{\rho^{\gamma}} .
$$

This relation establishes that $K$ remains constant in regions of the flow unaffected by dissipation, where $P \propto \rho^{\gamma}$.

If the gas is in local thermodynamic equilibrium, then one can demonstrate that the value of $K$ is related to the entropy per particle $S$ by (Reif 1965)

$$
S=k \ln K+c_{0},
$$

where $c_{0}$ is a constant that depends only the composition of the gas, but is independent of its state. Becker \& Le (2003) established that the asymptotic behaviors of $K(r)$ and $\ell(r)$ close to the event horizon are given by

$$
K(r)=K_{0}\left[1-\frac{16 \alpha \ell_{0}^{2}}{5 c^{2} r_{\mathrm{s}}^{5}}\left(\frac{r_{\mathrm{s}}}{2}\right)^{1 / 2}\left(r-r_{\mathrm{s}}\right)^{5 / 2}\right], \quad r \rightarrow r_{\mathrm{s}},
$$

and

$\ell(r)=\ell_{0}+\frac{2 \alpha \ell_{0}}{c^{2} r_{\mathrm{s}}}\left(\frac{2}{r_{\mathrm{s}}}\right)^{1 / 2}\left(\frac{K_{0}^{2}}{2 r_{\mathrm{s}}^{3}}\right)^{\frac{\gamma-1}{\gamma+1}}\left(r-r_{\mathrm{s}}\right)^{\frac{\gamma+5}{2 \gamma+2}}, \quad r \rightarrow r_{\mathrm{s}}$,

where $K_{0}$ represents the entropy value at the horizon. Note that the asymptotic radial dependence of $K(r)$ is very weak, reflecting the unimportance of viscous dissipation near the horizon. Becker \& Le (2003) also found that the asymptotic variation of the inflow velocity is given by

$v(r)=v_{\mathrm{ff}}(r)\left[1+\frac{2 \epsilon_{0} r^{2}-\ell_{0}^{2}-(\gamma+1) f(r)}{r^{2} v_{\mathrm{ff}}^{2}(r)-(\gamma-1) f(r)}\right]^{1 / 2}, \quad r \rightarrow r_{\mathrm{s}}$, 
where $\epsilon_{0}$ denotes the value of the specific energy transport rate at the horizon, the free-fall velocity is given by

$$
v_{\mathrm{ff}}(r)=\left(\frac{2 G M}{r-r_{\mathrm{s}}}\right)^{1 / 2},
$$

and the function $f$ is defined by

$$
f(r) \equiv \frac{2 \gamma r^{2}}{\gamma^{2}-1}\left[\frac{K_{0}^{2}}{2 r^{3}\left(r-r_{\mathrm{s}}\right)}\right]^{\frac{\gamma-1}{\gamma+1}} .
$$

For given values of the parameters $\epsilon_{0}, \ell_{0}$, and $K_{0}$, Equations (30) and (31) can be used to compute starting values for the integration of the variables $\ell(r)$ and $v(r)$ away from the event horizon.

\section{GLOBAL SOLUTIONS}

The structure of the global disk/shock model depends on the energy transport rate at the horizon $\epsilon_{0}$, the angular momentum at the horizon $\ell_{0}$, the entropy at the horizon $K_{0}$, the viscosity parameter $\alpha$, and the ratio of specific heats $\gamma$. In the calculations performed here, we set $\gamma=1.5$, following Narayan et al. (1997). When a shock is present, we use the subscripts "-" and "+" to denote quantities measured just upstream and just downstream from the shock, respectively. In a shocked disk, the gas passes through one critical point in the pre-shock region at $r=r_{c}^{\text {out }}$, and then through another in the post-shock region at $r=r_{c}^{\text {in }}$. Conversely, if the flow is smooth (shock-free), then the gas only passes through one critical point, located at radius $r=r_{c}$.

The process of determining the structure of a viscous ADAF disk containing an isothermal shock begins with the selection of input values for $\epsilon_{0}, \ell_{0}, \alpha$, and $\gamma$. We must also select a provisional value for the entropy parameter $K_{0}$. Based on this information, we can utilize the asymptotic relations given by Equations (30) and (31) to compute values for $\ell(r)$ and $v(r)$, respectively, at the starting radius for the outward integration, which is $2.001 G M / c^{2}$. Next we numerically integrate Equations (11), (15), and (20), beginning at the starting radius and working outward toward the inner critical point. The value of $K_{0}$ is varied until a solution is obtained that satisfies the critical conditions given by Equations (24) and (25), which ensure that the flow passes smoothly through the inner critical point at radius $r_{c}^{\text {in }}$.

After the location of the inner critical point has been established, the integration must be continued into the upstream, subsonic region. Due to the mathematical structure of the dynamical Equation (21), it is not possible to integrate across the critical point itself. We must therefore employ linear extrapolation, which requires the calculation of the derivative $d v / d r$ at the critical point using l'Hôpital's rule. After performing the linear extrapolation, the integration is continued in the subsonic region until we reach the shock radius, $r_{*}$, which is assigned a provisional value initially. Since no energy is lost from the disk between the shock and the horizon, it follows that $\epsilon_{0}=\epsilon_{+}$, where $\epsilon_{+}$is the energy transport rate on the downstream side of the shock. At the shock location, the velocity and the energy transport rate experience discontinuous transitions described by the isothermal shock jump conditions, which can be written as (e.g., Chakrabarti 1989a)

$$
\frac{v_{+}}{v_{-}}=\mathcal{M}_{+}^{2} \quad \Delta \epsilon \equiv \epsilon_{+}-\epsilon_{-}=\frac{v_{+}^{2}-v_{-}^{2}}{2},
$$

where $\mathcal{M}_{+} \equiv v_{+} / a_{+}$denotes the post-shock Mach number. This relation facilitates the calculation of the pre-shock (incident)

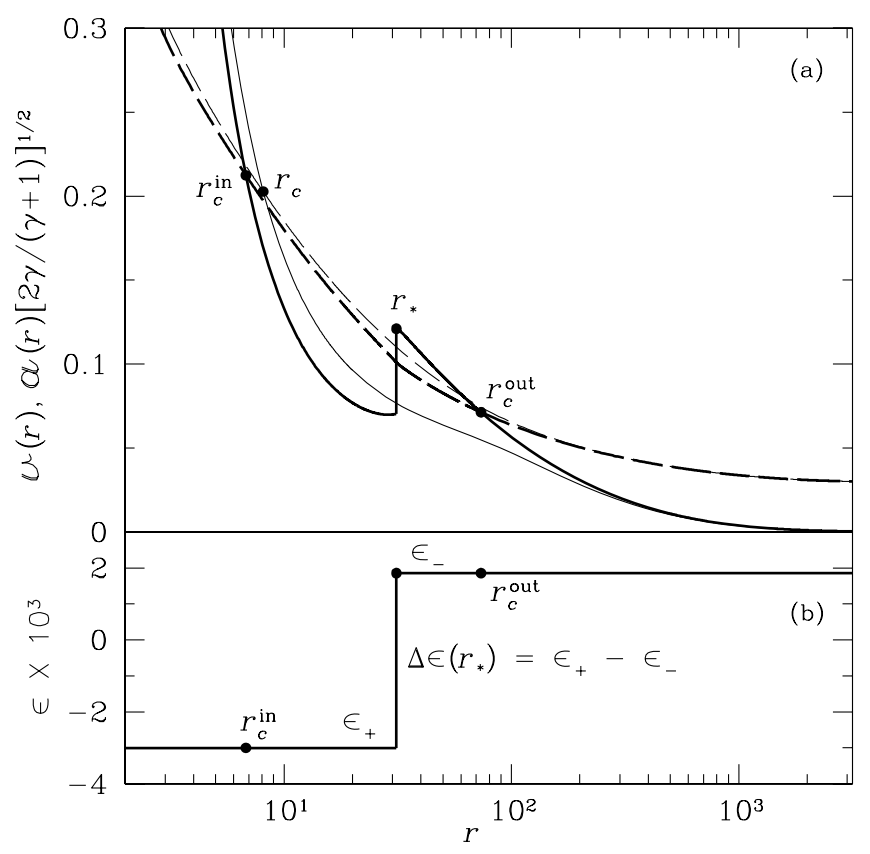

Figure 1. (a) Inflow velocity $v$ (solid lines) and isothermal sound speed $a$ multiplied by $\sqrt{2 \gamma /(\gamma+1)}$ (dashed lines) plotted as functions of the radius $r$ in units of $G M / c^{2}$ for a typical global accretion solution with $\gamma=1.5$. The thick and thin lines represent the shocked and smooth solutions, respectively. (b) Energy transport rate $\epsilon$ plotted as a function of radius $r$. Note the jump to the negative post-shock value due to the release of energy through the upper and lower surfaces of the accretion disk at the isothermal shock location. See the text for details.

energy transport rate, $\epsilon_{-}$. Note that if no shock is present, then $\epsilon$ is continuous throughout the entire flow, and therefore $\Delta \epsilon=0$ and $\epsilon_{0}=\epsilon_{-}$. By definition, the flow maintains a uniform temperature across the isothermal shock, and consequently $a_{+}=a_{-}$. The shock radius $r_{*}$ is varied until we obtain a solution that passes smoothly through the outer critical point, located in the upstream region at radius $r_{c}^{\text {out }}$. The solution process is completed when the critical conditions are satisfied at the outer critical point. For given input values of $\epsilon_{0}, \ell_{0}, \alpha$, and $\gamma$, in the end we obtain unique values for $K_{0}, r_{*}, r_{c}^{\text {in }}$, and $r_{c}^{\text {out }}$, in addition to the associated global dynamical solution for the disk/shock structure.

A typical set of shocked and smooth accretion solutions obtained for the same value of the incident energy transport rate $\epsilon_{-}$is presented in Figure 1(a). In these calculations, the ShakuraSunyaev viscosity parameter was specified by setting $\alpha=0.1$, and the specific heat ratio is given by $\gamma=1.5$. By applying the multi-step iterative method described above, we obtain the parameters $\epsilon_{-}=0.001856, \epsilon_{0}=\epsilon_{+}=-0.003, \ell_{0}=2.55$, and $K_{0}=0.00689$ for the shocked flow. In the shocked case, the incident flow first becomes supersonic upon passing through the outer critical point located at $r_{c}^{\text {out }}=73.51$. The gas subsequently passes through an isothermal shock at $r_{*}=31.2$, where the velocity makes a discontinuous transition to become subsonic again. Downstream from the shock, the flow accelerates towards the horizon, and regains its supersonic character after crossing the inner critical point at $r_{c}^{\text {in }}=6.798$. The radius at the outer edge of the hot region, $r_{\text {edge }}=3555$, is computed by setting $\ell=\ell_{\mathrm{K}}$, so that the angular momentum matches the local Keplerian value. The radial profile of the specific angular momentum $\ell$ is plotted in Figure 2(b). Although the incident energy transport rate $\epsilon_{-}$is negative in our calculations, it should be emphasized that the total energy inflow rate, including the rest mass energy, is positive as required. 


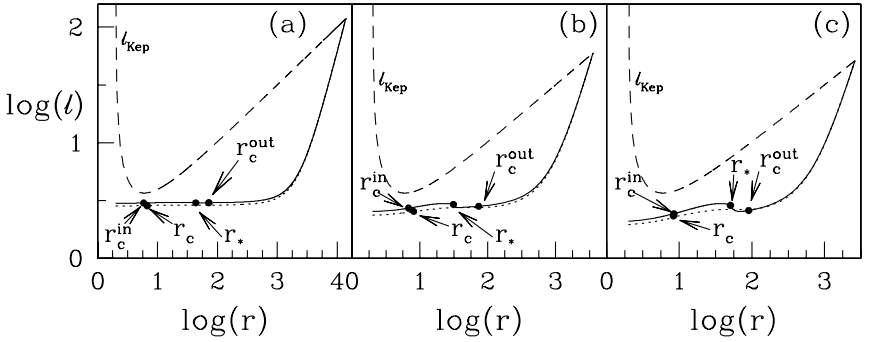

Figure 2. Specific angular momentum $\ell$ plotted as a function of the radius $r$ for (a) $\alpha=0.01$, (b) $\alpha=0.1$, and (c) $\alpha=0.2$. Here we assume that $\gamma=1.5$. The solid, dotted, and dashed lines correspond to the shocked, smooth, and Keplerian profiles, respectively. See the text for the complete sets of parameters used in each panel.

For the smooth-flow solution depicted in Figure 1(a), we used the parameters $\epsilon_{0}=\epsilon_{+}=\epsilon_{-}=0.001856, \ell_{0}=2.368$, and $K_{0}=0.008565$. In this case, the value of $\ell_{0}$ was selected so that $\ell=\ell_{\mathrm{K}}$ at the same outer edge radius, $r_{\text {edge }}=3555$, obtained in the shocked disk (see Figure 2(b)). The smooth flow solution passes through a single critical point, located at $r_{c}=8.097$. It is interesting to compare the plots of the sound speed obtained in the smooth and shocked cases. The loss of energy at the shock significantly reduces the sound speed (and therefore the temperature) in the post-shock region relative to the smooth solution. In Figure 1(b), we plot the variation of the energy transport rate for the shock solution. The excess energy released through the upper and lower surfaces of the disk at the shock location may be sufficient to power the formation of a relativistic jet (Le \& Becker 2005; Becker et al. 2008).

The viscosity parameter $\alpha$ plays a central role in determining the efficiency of the angular momentum transport in the disk. We explore the effect of varying this parameter in Figure 2, where we plot the results obtained for the radial profile of the specific angular momentum $\ell$ in both shocked and smooth disks for three different values of $\alpha$. All of the solutions are sub-Keplerian $\left(\ell<\ell_{\mathrm{K}}\right)$ for $r<r_{\text {edge. }}$. In the shocked case, the increase in the angular momentum in the post-shock region produces the "centrifugal barrier" required for shock formation, while no such barrier exists in the smooth case. In each of the panels, the value of $\ell_{0}$ was determined by satisfying the inner critical conditions, and the incident energy transport rate $\epsilon_{-}$has the same value in the shocked and smooth scenarios. The parameter values adopted in panel (a) are $\alpha=0.01, \gamma=1.5, \epsilon_{+}=-1.0 \times 10^{-3}$, $\epsilon_{-}=1.9072 \times 10^{-3}, \ell_{0}=3.0, K_{0}=2.7509 \times 10^{-3}$ in the shocked case, and $\epsilon_{+}=\epsilon_{-}=1.9072 \times 10^{-3}, \ell_{0}=2.845$, $K_{0}=3.8588 \times 10^{-3}$ in the smooth (shock-free) case. In panel (b), we adopt the same parameters used in Figure 1, namely $\alpha=0.1, \gamma=1.5, \epsilon_{+}=-3.0 \times 10^{-3}, \epsilon_{-}=1.856 \times 10^{-3}$, $\ell_{0}=2.55, K_{0}=6.89 \times 10^{-3}$ in the shocked case, and $\epsilon_{+}=\epsilon_{-}=1.856 \times 10^{-3}, \ell_{0}=2.368, K_{0}=8.565 \times 10^{-3}$ in the smooth case. The values adopted in panel (c) are $\alpha=0.2, \gamma=1.5, \epsilon_{+}=-1.0 \times 10^{-3}, \epsilon_{-}=1.6483 \times 10^{-3}$, $\ell_{0}=2.1, K_{0}=1.1883 \times 10^{-2}$ in the shocked case, and $\epsilon_{+}=\epsilon_{-}=1.6483 \times 10^{-3}, \ell_{0}=1.966, K_{0}=1.25 \times 10^{-2}$ in the smooth case. Note the increasing prominence of the centrifugal barrier as $\alpha$ increases.

\section{SHOCK PROPERTIES}

Since this is the first work to extensively consider the consequences of a standing shock for the structure of a viscous accretion disk, it is interesting to examine how the shock properties depend on the various flow parameters. We carry

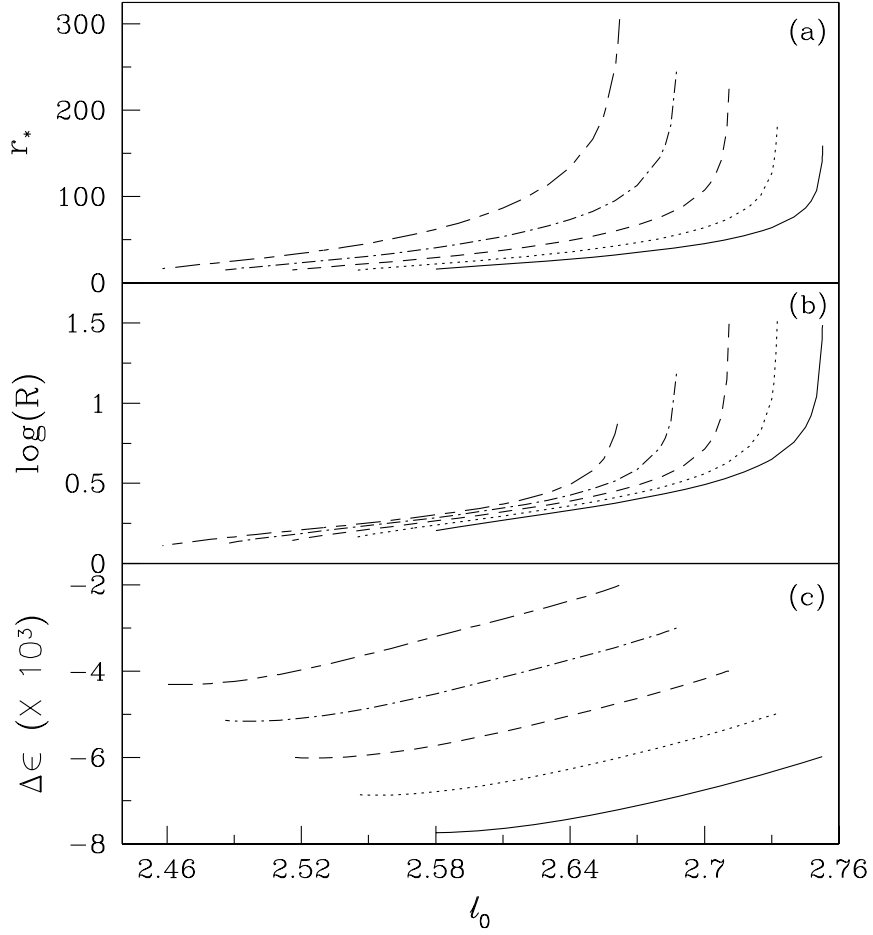

Figure 3. Variation of (a) the isothermal shock radius $r_{*}$, (b) the compression ratio $R=v_{-} / v_{+}$, and (c) the energy jump $\Delta \epsilon$ as functions of $\ell_{0}$. The solid, dotted, dashed, dot-dashed, and short-long-dashed curves correspond to $\epsilon_{0}=-0.006,-0.005,-0.004,-0.003$, and -0.002 , respectively. Here, we set $\alpha=0.1$ and $\gamma=1.5$.

out the analysis for flows characterized by the fundamental parameters $\left(\epsilon_{0}, \ell_{0}, \alpha\right)$, where $\epsilon_{0}$ denotes the energy transport rate at the horizon, which is the same as the post-shock energy transport rate $\epsilon_{+}$. In each case, we keep one of the three parameters $\left(\epsilon_{0}, \ell_{0}, \alpha\right)$ fixed and vary the other two to study the resulting shock properties.

In Figure 3, we present the results obtained for the shock radius, $r_{*}$, the compression ratio, $R=v_{-} / v_{+}$, and the energy jump, $\Delta \epsilon=\epsilon_{+}-\epsilon_{-}$, as functions of the angular momentum transport rate $\ell_{0}$ and the energy transport rate at the horizon $\epsilon_{0}$. In these calculations, the viscosity and specific heat parameters are held constant, with $\alpha=0.1$ and $\gamma=1.5$, respectively. Figure 3(a) clearly demonstrates that accretion flows with a very wide range of input parameters may possess isothermal standing shocks. Note that for a given post-shock energy parameter $\epsilon_{0}$, the shock location recedes away from the black hole with increasing $\ell_{0}$ because of the enhancement of the centrifugal barrier. On the other hand, the shock location shifts inward as $\epsilon_{0}$ decreases for a given value of $\ell_{0}$, due to the increased ram pressure of the flow. For a given value of $\epsilon_{0}$, there is a restricted range of $\ell_{0}$ values within which a shock can exist. In general, we find that the shock radius $r_{*}$ always exceeds $\sim 10 G M / c^{2}$. In Figure 3(b), we plot the variation of the shock compression ratio $R$ as a function of the flow parameters $\epsilon_{0}$ and $\ell_{0}$. We observe that for fixed $\epsilon_{0}$, the compression ratio increases as the shock moves away from the black hole. In Figure 3(c), we plot the corresponding variation of the energy jump $\Delta \epsilon$. Note that $\Delta \epsilon$ becomes more negative as $\epsilon_{0}$ decreases for fixed $\ell_{0}$, which reflects the fact that a larger amount of gravitational potential energy is released into the outflow when the shock wave forms closer to the black hole.

Next we examine the dependence of the shock properties on the viscosity parameter $\alpha$. In Figure 4(a), the isothermal shock location $r_{*}$ is plotted as a function of $\alpha$ for various values of the 


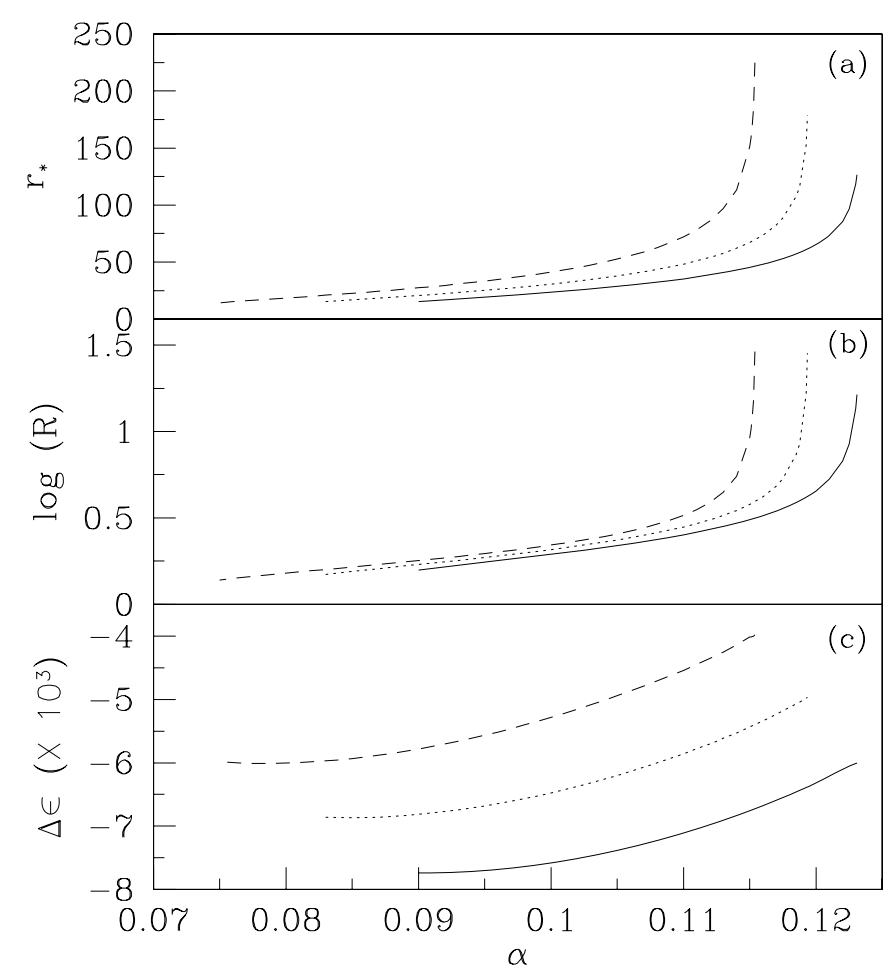

Figure 4. Variation of (a) the isothermal shock radius $r_{*}$, (b) the compression ratio $R=v_{-} / v_{+}$, and (c) the energy jump $\Delta \epsilon$ as functions of $\alpha$. The solid, dotted, and dashed lines denote $\epsilon_{0}=-0.006,-0.005$, and -0.004 , respectively. Here, we set $\ell_{0}=2.62$ and $\gamma=1.5$.

post-shock (horizon) energy transport rate $\epsilon_{0}$ for the case with $\gamma=1.5$. Here, we fix the angular momentum transport rate by setting $\ell_{0}=2.62$. In all cases, we observe that shocks can form over a wide range of flow parameters. For a given $\alpha$, the shock tends to form farther away from the black hole as $\epsilon_{0}$ is increased, which is consistent with the behavior noted in Figure 3(a). We also observe that for fixed $\epsilon_{0}$, the shock radius $r_{*}$ increases with $\alpha$. This is because higher values of $\alpha$ tend to reduce the angular momentum, which forces the centrifugal barrier to operate at a larger radius in order to be effective. Note that for a given set of flow parameters $\left(\epsilon_{0}, \ell_{0}\right)$, the viscosity parameter $\alpha$ has both upper and lower limits for shock formation. The lower limit reflects the fact that fully inviscid flows cannot form shocks for arbitrary combinations of $\epsilon_{0}$ and $\ell_{0}$ (e.g., Le \& Becker 2005). Conversely, the upper limit on $\alpha$ is due to the efficient dissipation of angular momentum that occurs as $\alpha$ increases. This effect prevents the development of the centrifugal barrier required for shock formation if $\alpha$ is too large.

The dependence of the shock properties on the post-shock energy $\epsilon_{0}$ is illustrated in Figure 5 for three values of the angular momentum transport rate $\ell_{0}$. The viscosity and specific heat parameters are held constant with the values $\alpha=0.1$ and $\gamma=1.5$, respectively. In Figure 5(a) we plot the shock location $r_{*}$ as a function of $\epsilon_{0}$. Since the standing shock is centrifugally supported, the shock radius $r_{*}$ increases when $\ell_{0}$ is increased for a fixed value of $\epsilon_{0}$. On the other hand, if we hold $r_{*}$ constant, then we observe an anticorrelation between $\ell_{0}$ and $\epsilon_{0}$. In Figures 5(b) and (c), respectively, we depict the associated variations of the compression ratio $R$ and the energy jump $\Delta \epsilon$ as functions of $\epsilon_{0}$ for the same set of flow parameters used in Figure 5(a). The compression ratio increases when either $\ell_{0}$ or $\epsilon_{0}$ is increased. We note that $\Delta \epsilon$ increases almost linearly with increasing $\epsilon_{0}$ for fixed $\ell_{0}$. The results indicate that flows with larger values of $\ell_{0}$ tend to discharge less energy at the shock location.

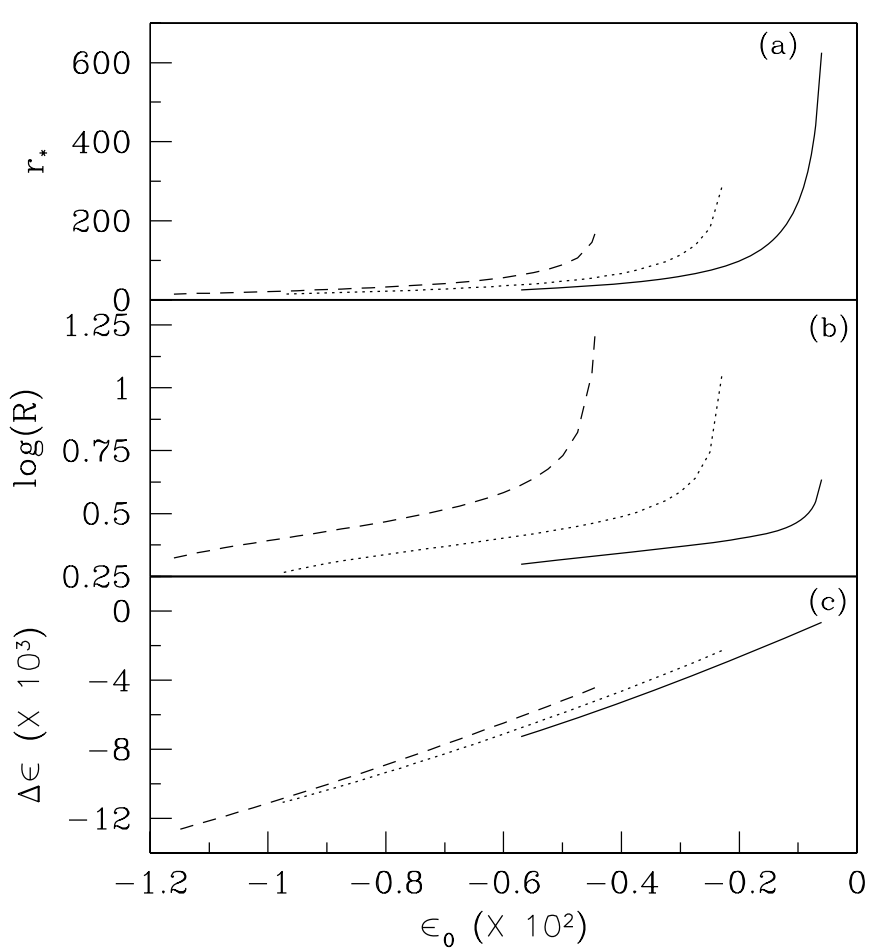

Figure 5. Variation of (a) the isothermal shock radius $r_{*}$, (b) the compression ratio $R=v_{-} / v_{+}$, and (c) the energy jump $\Delta \epsilon$ as functions of $\epsilon_{0}$. The solid, dotted, and dashed curves represent $\ell_{0}=2.62,2.67$, and 2.72 , respectively. Here, we set $\alpha=0.1$ and $\gamma=1.5$.

The hot, sub-Keplerian ADAF region must connect with a cool Keplerian outer disk at some large radius. We estimate the outer edge radius of the ADAF zone, $r_{\text {edge }}$, by setting the angular momentum of the accretion flow equal to the local Keplerian value. In Figure 6 , we plot the variation of the outer edge radius as a function of $\ell_{0}$ and $\epsilon_{0}$ for the case with $\alpha=0.1$ and $\gamma=1.5$. We observe that for a fixed value of $\epsilon_{0}$, the value of $r_{\text {edge }}$ rapidly increases with increasing $\ell_{0}$, indicating that the cool Keplerian disk recedes from the horizon. The outer edge radius also increases with increasing $\epsilon_{0}$ when $\ell_{0}$ is held fixed.

The shocked, viscous, advection-dominated disks discussed here represent a new type of astrophysical phenomenon, and therefore it is important to investigate the range of flow parameters within which shocks can form. In Figure 7, we analyze this question for the case with $\gamma=1.5$ by varying the angular momentum transport rate $\ell_{0}$ and the upstream (incident) energy transport rate $\epsilon_{-}$while holding the viscosity parameter $\alpha$ fixed at five different values. The solid curve depicts the region within which shocked solutions are possible for fully inviscid flow $(\alpha=0)$. This result agrees very well with Figure 2 from Le $\&$ Becker (2005). We note that the allowed region for the shocked solution shifts toward lower angular momentum as $\alpha$ increases. This shift occurs because the specific angular momentum of the accreting material at the horizon, $\ell_{0}$, decreases in response to the enhanced viscosity associated with the increase in $\alpha$. The shrinkage of the region for the shocked solution observed as $\alpha$ increases suggests that shocked disk solutions are impossible above a critical value of $\alpha$, denoted by $\alpha_{\text {crit }}$. For the model considered here, with $\gamma=1.5$, we find that $\alpha_{\text {crit }} \approx 0.27$.

\section{ASTROPHYSICAL APPLICATIONS}

It is interesting to ask whether the self-consistent disk/ shock model we have described can account for the energetics 


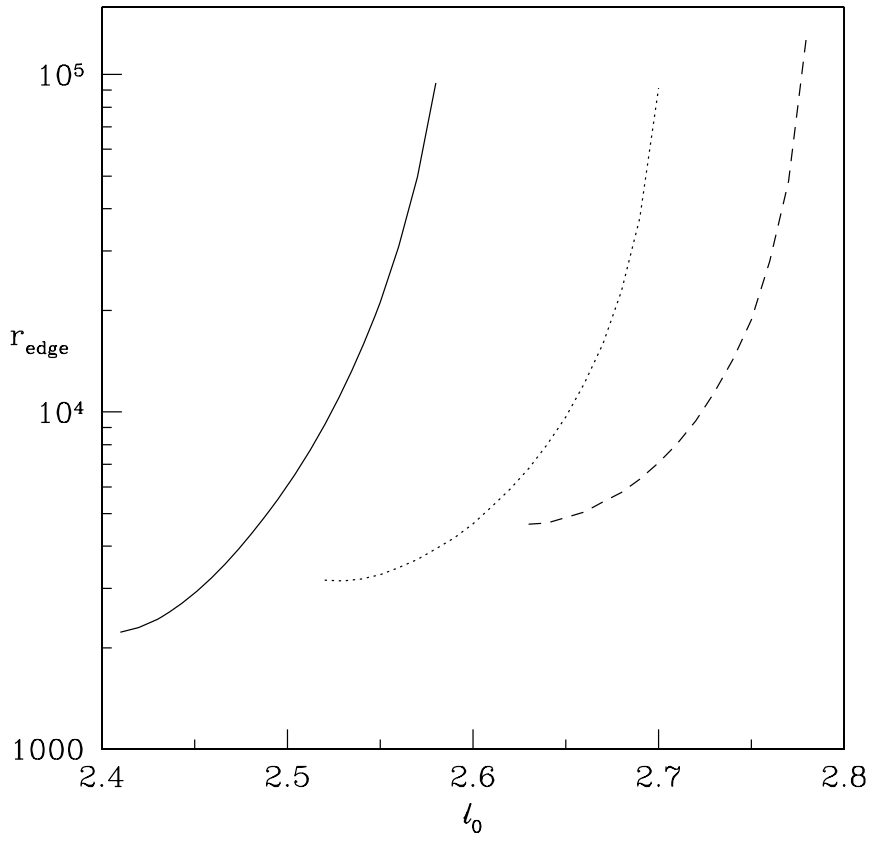

Figure 6. Plot of the outer edge radius $r_{\text {edge }}$ as a function of $\ell_{0}$. This is the radius where the angular momentum $\ell$ equals the Keplerian value $\ell_{\mathrm{K}}$ (Equation (12)). The solid, dotted, and dashed lines correspond to $\epsilon_{0}=0.0$, -0.004 , and -0.008 , respectively. Here, we set $\alpha=0.1$ and $\gamma=1.5$. See the text for details.

$$
\times 10^{-3}
$$

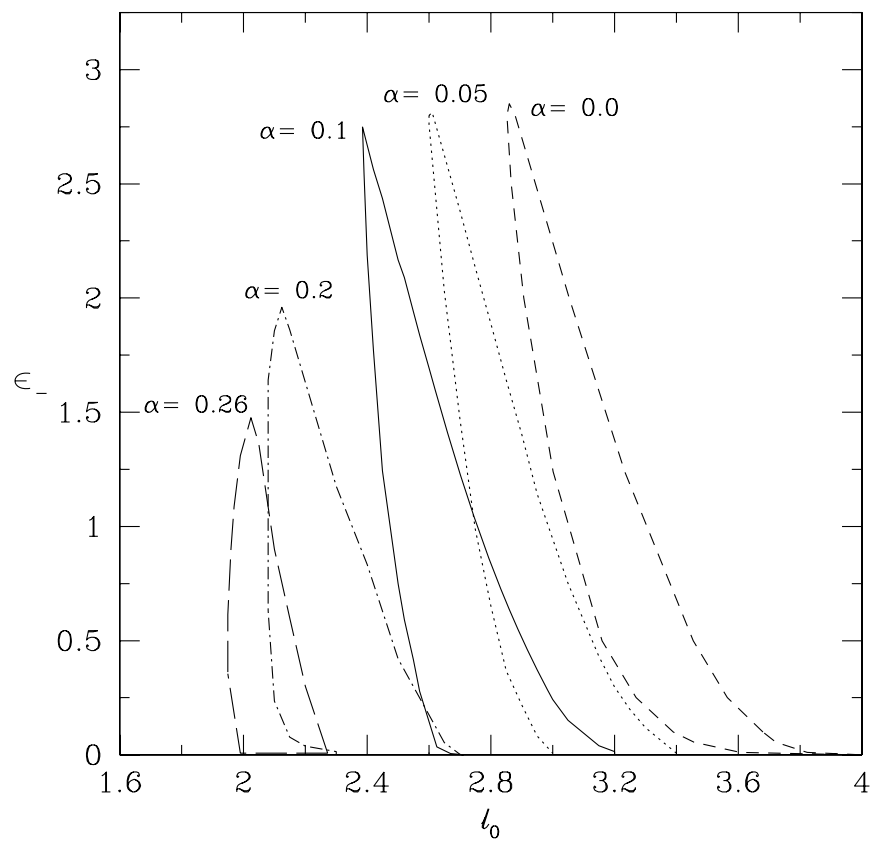

Figure 7. Representation of the parameter space spanned by the specific angular momentum at the horizon $\ell_{0}$ and the incident energy transport rate $\epsilon_{-}$when $\gamma=1.5$. The wedge-shaped regions denote the areas of the parameter space within which shocks can form for the indicated values of $\alpha$. See the discussion in the text.

of specific jet sources, such as M87 and Sgr A*. When observational values of the black hole mass $M$, the accretion rate $\dot{M}$, and the jet kinetic luminosity $L_{\text {jet }}$ are available for a particular source, we can utilize this information to constrain the model parameters. The process begins by selecting values for the fundamental free parameters $\epsilon_{0}, \alpha$, and $\gamma$. After selecting provisional values for $\ell_{0}$ and $K_{0}$, we utilize the asymptotic relations given by Equations (30) and (31) to compute initial values for the functions $\ell(r)$ and $v(r)$ at the starting radius $2.001 G M / c^{2}$. We then numerically integrate Equations (11), (15), and (20) in the outward direction, and vary the value of $K_{0}$ until the critical conditions given by Equations (24) and (25) are satisfied at the inner critical point.

The energy transport rate $\epsilon$ drops from its upstream value $\epsilon_{-}$ to the downstream value $\epsilon_{+}=\epsilon_{0}$ at the shock location due to the escape of energy from the disk into the outflow (jet). We can therefore determine the shock radius $r_{*}$ by requiring that the value of $\Delta \epsilon$ computed using Equation (34) agrees with that calculated based on the observed jet kinetic luminosity, $L_{\mathrm{jet}}$, using the relation

$$
L_{\text {jet }}=-\dot{M} \Delta \epsilon,
$$

where the negative sign appears because $\Delta \epsilon<0$. Once the shock radius is established, the integration is continued on the upstream side of the shock toward the outer critical point. If the flow does not pass smoothly through the outer critical point, then the value of $\ell_{0}$ is modified and the procedure is repeated starting from the horizon. The computation of the selfconsistent disk/shock dynamical model is complete when the critical conditions are satisfied at the outer critical point. For given input values of $\epsilon_{0}, \alpha$, and $\gamma$, in the end we obtain the complete global dynamical solution along with unique values for $\ell_{0}, K_{0}, r_{*}, r_{c}^{\text {in }}$, and $r_{c}^{\text {out }}$. We provide two specific examples below.

\section{1. $M 87$}

The disk/shock/outflow model we have developed was previously utilized by Becker et al. (2008) to analyze the structure of the accretion flow in M87. The results obtained for that source are presented in more detail here. Our application to M87 is based on the observational values $\dot{M}=1.34 \times 10^{-1} M_{\odot} \mathrm{yr}^{-1}$ (Reynolds et al. 1996), $L_{\text {jet }}=5.5 \times 10^{43} \mathrm{erg} \mathrm{s}^{-1}$ (Reynolds et al. 1996; Bicknell \& Begelman 1996; Owen et al. 2000), and $M=3.0 \times 10^{9} M_{\odot}$ (e.g., Ford et al. 1994). We set the viscosity parameter $\alpha=0.1$ in order to demonstrate that shocks can exist in ADAF disks even in the presence of substantial viscosity, although our model can accommodate any value for $\alpha$. Based on the assumption of approximate energy equipartition between the magnetic field and the gas internal energy, we adopt the value $\gamma=1.5$ for the adiabatic index (Narayan et al. 1997). We utilize natural gravitational units in our numerical examples, with $G M=c=1$ and $r_{\mathrm{s}}=2$, except as noted. The remaining parameters for the shocked-disk model can be computed based on the observations of M87, from which we obtain $\epsilon_{-}=0.001516, \epsilon_{+}=\epsilon_{0}=-0.005746, \ell_{0}=2.6257$, $K_{0}=0.00608, r_{*}=26.329, r_{c}^{\text {in }}=6.462$, and $r_{c}^{\text {out }}=96.798$. For the pre-shock and post-shock velocities and Mach numbers we obtain $v_{-}=0.138, v_{+}=0.068, \mathcal{M}_{-}=1.427$, and $\mathcal{M}_{+}=0.701$, respectively, and for the disk half-thickness at the shock location we obtain $H_{*}=12.10$.

The results obtained for the inflow speed $v$ and the isothermal sound speed $a$ are plotted in Figure 8(a), and the associated solution for the specific angular momentum $\ell$ is depicted along with the Keplerian profile $\ell_{\mathrm{K}}$ (Equation (12)) in Figure 8(b). Results are presented for both shocked and smooth (shock-free) disks. In the shocked disk, the source is located at the shock radius, $r_{*}=26.329$, and we observe that the values of $\ell$ and $\ell_{\mathrm{K}}$ merge at $r_{\text {edge }}=4658$, which represents the outer edge of the ADAF region. The smooth solution is computed by integrating the conservation equations using the same value for the incident energy transport rate $\epsilon_{-}$as adopted in the shocked-disk model, 


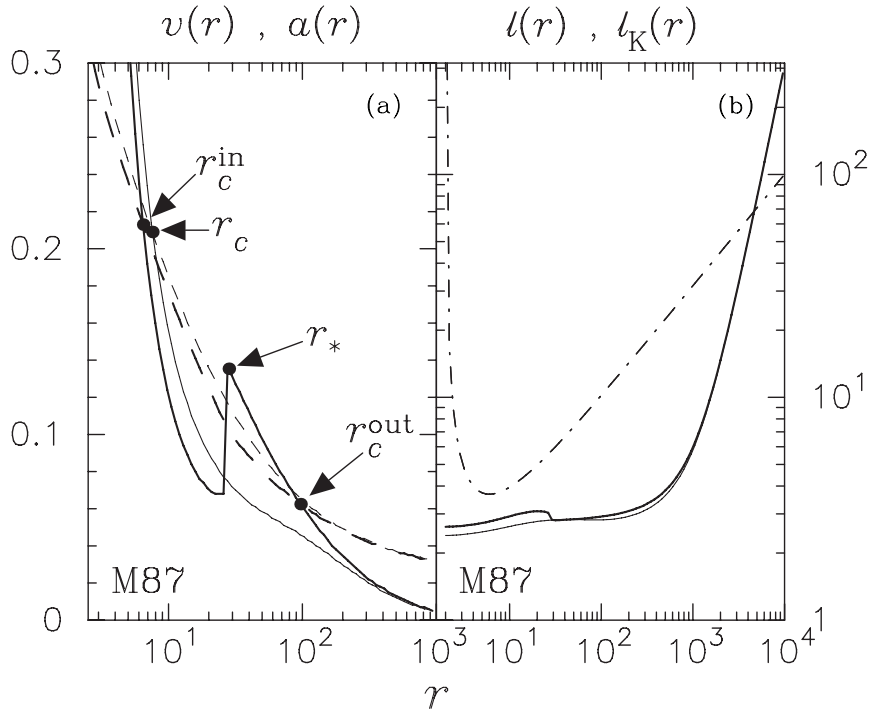

Figure 8. Dynamical profiles for M87. (a) Inflow velocity $v$ (solid lines) and isothermal sound speed $a$ multiplied by $\sqrt{2 \gamma /(\gamma+1)}$ (dashed lines) plotted as functions of the radius $r$ in units of $G M / c^{2}$. The thick and thin lines denote the shocked and smooth solutions, respectively. (b) Specific angular momentum $\ell$ for the shocked (thick line) and smooth (thin line) solutions plotted as functions of $r$ in units of $G M / c^{2}$ along with the Keplerian angular momentum $\ell_{\mathrm{K}}$ (dotdashed line).

but with no shock included. The value of $\ell_{0}$ is then varied until we obtain $\ell=\ell_{\mathrm{K}}$ at the same outer radius, $r_{\text {edge }}=4658$, as in the shocked disk. The resulting parameter values for the smooth solution are $\epsilon_{-}=\epsilon_{+}=0.001516, \ell_{0}=2.3988, K_{0}=0.0084$, and $r_{c}=7.572$. In order to facilitate comparison with the shocked model, in the smooth case the source is assumed to be at the same radius $r=26.329$.

Our solutions for the specific angular momentum $\ell$ are significantly sub-Keplerian, in agreement with the results obtained by Narayan et al. (1997), who utilized the same set of ADAF conservation equations we employ. The sub-Keplerian nature of the flow stems from the relatively high value for the viscosity parameter adopted here, $\alpha=0.1$. Note that the sound speed (and hence the temperature) is significantly lower in the shocked case as compared with the smooth disk due to the release of energy into the outflow at the shock location.

It is important to examine the energy balance in the shocked model to ensure that the rate of energy loss at the shock location is equal to the observational value for the kinetic luminosity in M87. We can compute the theoretical value for the jet luminosity, $L_{\text {jet }}$, by substituting our values for $\epsilon_{+}$and $\epsilon_{-}$into Equation (35). The result obtained is $L_{\text {jet }}=5.5 \times 10^{43} \mathrm{erg} \mathrm{s}^{-1}$, which agrees with the observed jet luminosity for M87. This confirms that our viscous shocked disk model is able to account for the energetics of the M87 outflow.

\section{2. $\operatorname{Sgr} A^{*}$}

For Sgr A*, we adopt the values $M=2.6 \times 10^{6} M_{\odot}$ (Schödel et al. 2002) and $\dot{M}=8.96 \times 10^{-7} M_{\odot} \mathrm{yr}^{-1}$ (Yuan et al. 2002; Quataert 2003), and we set $\gamma=1.5$ and $\alpha=0.1$. The kinetic luminosity of the jet is estimated using $L_{\text {jet }}=$ $5 \times 10^{38} \mathrm{erg} \mathrm{s}^{-1}$ (Falcke \& Biermann 1999), although this value is rather uncertain (e.g., Yuan 2000; Yuan et al. 2002). The results for the remaining model parameters implied by the observations are $\epsilon_{-}=0.00134884, \epsilon_{+}=\epsilon_{0}=-0.0085$, $\ell_{0}=2.6728, K_{0}=0.005448, r_{*}=19.917, r_{c}^{\text {in }}=6.380$, and $r_{c}^{\text {out }}=112.384$. For the pre-shock and post-shock velocities

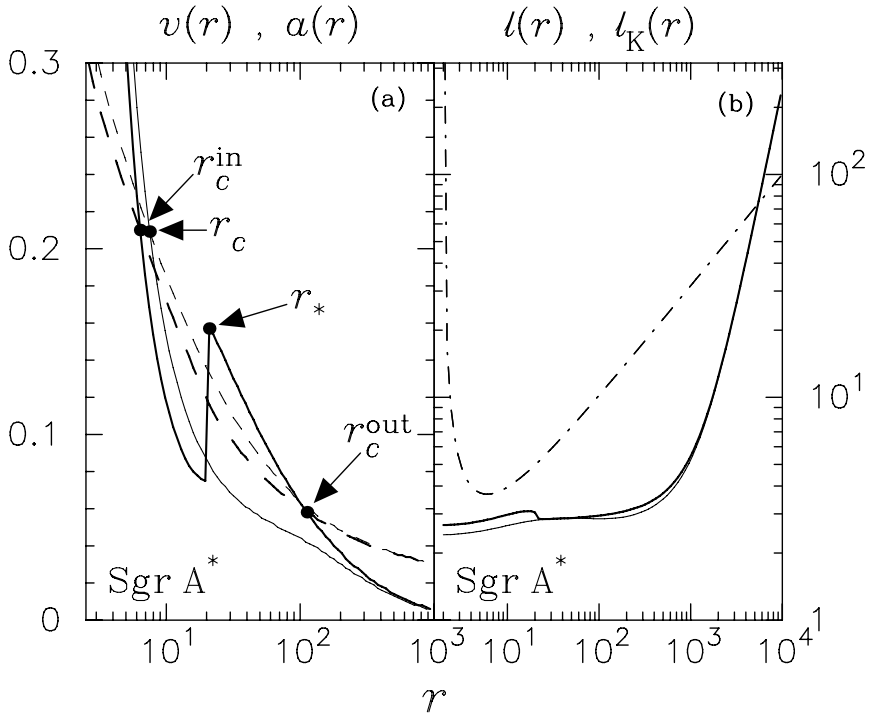

Figure 9. Same as Figure 8, except model results are for Sgr A*.

and Mach numbers we obtain $v_{-}=0.159, v_{+}=0.0748$, $\mathcal{M}_{-}=1.4578$, and $\mathcal{M}_{+}=0.6860$, respectively, and for the disk half-thickness at the shock location we obtain $H_{*}=8.72$.

In Figure 9(a), we plot the results obtained for the inflow speed $v$ and the isothermal sound speed $a$ in both the shocked and smooth disk models for $\operatorname{Sgr} \mathrm{A}^{*}$. The profiles of $\ell$ and $\ell_{\mathrm{K}}$ are plotted in Figure 9(b). In the shocked case, the source is located at $r_{*}=19.917$, and we find that $\ell=\ell_{\mathrm{K}}$ at radius $r_{\text {edge }}=5432$, which is the outer edge of the ADAF region. The incident energy transport rate $\epsilon_{-}=0.00134884$ is the same for both the smooth and shocked models, and the value of $\ell_{0}$ in the smooth case is determined by requiring that $\ell$ merges with the Keplerian profile at the same radius $r_{\text {edge }}=5432$ as in the shocked case. Based on this approach, the parameters obtained for the smooth model are $\epsilon_{-}=\epsilon_{+}=0.00134884, \ell_{0}=2.416, K_{0}=0.008293$, and $r_{c}=$ 7.511. We assume for consistency that the source is located at the same radius $r=19.917$ in both the smooth and shocked cases.

The results for the specific angular momentum $\ell$ are significantly sub-Keplerian, as expected based on the relatively high value utilized for the viscosity parameter, $\alpha=0.1$ (cf. Figure $8(\mathrm{~b})$ ). The sound speed is lower when a shock is present, due to the escape of energy at the shock location, as noted previously in Figure 8(a). The result for $L_{\text {jet }}$ obtained by substituting our values for $\epsilon_{+}$and $\epsilon_{-}$into Equation (35) is equal to the observed jet luminosity, $5 \times 10^{38} \mathrm{erg} \mathrm{s}^{-1}$, and this establishes that our shocked disk model successfully accounts for the observed energetics of the accretion flow and the outflow in Sgr A*.

\section{CONCLUSION}

In this paper we have presented the first systematic study of the structure of stationary, advection-dominated, shocked viscous accretion disks around black holes. Our primary focus here has been on the global properties of accretion flows containing isothermal shock waves, formed as a result of the presence of a centrifugal barrier located near the event horizon. Our model is based on the same set of standard ADAF conservation equations considered by Narayan et al. (1997). The conservation equations are supplemented by the inner boundary conditions developed by Becker \& Le (2003) which allow the calculation of starting values for the physical variables close to the horizon. Once the starting values are established, a stable outward integration method is employed to obtain the radial 
profiles of the various flow quantities such as velocity, sound speed, and angular velocity. The fundamental model parameters are the energy inflow rate at the horizon, $\epsilon_{0}$, the specific angular momentum of the material at the horizon, $\ell_{0}$, the entropy parameter at the horizon, $K_{0}$, the Shakura-Sunyaev viscosity parameter, $\alpha$, and the ratio of specific heats, $\gamma$.

For given observational values of the black hole mass $M$, the accretion rate $\dot{M}$, and the jet kinetic luminosity $L_{\text {jet }}$, we are free to select values for $\alpha, \gamma$, and $\epsilon_{0}$. Following Narayan et al. (1997), we have set $\gamma=1.5$ in the calculations presented here. The remaining parameters $\ell_{0}$ and $K_{0}$, and the shock radius $r_{*}$, are subsequently determined by applying the asymptotic inner boundary conditions given by Equations (30) and (31), the critical conditions given by Equations (24) and (25), and the shock jump conditions given by Equations (34) and (35). The dynamical structure of the disk/shock system is obtained by numerically integrating Equations (11), (15), and (20) in the outward direction beginning at a starting radius located close to the event horizon.

The formation of a shock requires that the flow possesses at least two critical points. The existence of multiple critical points in both adiabatic and polytropic accretion flows has been reported by many authors (e.g., Abramowicz \& Zurek 1981; Chakrabarti 1989a, 1989b, 1990, 1996). Flows with multiple critical points are confined to a restricted region of the parameter space, as indicated in Figure 7. Accretion flows within this region can form isothermal shocks. In their earlier study, Narayan et al. (1997) focused solely on solutions that pass only through the inner critical point, and consequently they did not obtain shocked disk solutions. Flows with discontinuities must first pass through an outer critical point, and subsequently through a shock and an inner critical point in order to represent physically acceptable accretion solutions. That is the scenario we have investigated here.

The possible presence of shock waves in accretion disks and their observational implications has been examined by a variety of previous authors. Abramowicz \& Chakrabarti (1990) presented one of the earliest studies of the properties of standing shock waves in accretion disks. Subsequently, the particular importance of isothermal shock waves was realized by Le \& Becker $(2004,2005,2007)$, who pointed out that efficient particle acceleration in the vicinity of a standing isothermal shock can help to explain the formation of the relativistic outflows of matter commonly observed around radio-loud, underfed black holes. However, all of these previous studies of isothermal shocks in accretion disks neglected the important role of viscosity in determining the disk structure. Very recently, Becker et al. (2008) demonstrated that first-order Fermi acceleration inside a viscous, shocked ADAF can accelerate particles more efficiently than in a smooth disk. Hence the presence of a standing shock wave can create a potentially favorable environment for the production of relativistic outflows. In this paper, we have presented a detailed study of the dynamics of viscous, shocked ADAF disks that are governed by the standard description of the angular momentum transport based on the Shakura-Sunyaev prescription for the kinematic viscosity. In particular, we confirm that the energetics of the viscous disk/shock model can explain the observational properties of both the accretion flow and the relativistic outflows in M87 and Sgr A*.

In Figure 7 we explored the parameter space within which ADAF disks with isothermal shocks can exist as a function of the specific angular momentum at the horizon $\ell_{0}$ and the incident energy transport rate $\epsilon_{-}$for various (constant) values of the viscosity parameter $\alpha$. The shock region shifts toward lower values of $\ell_{0}$ as $\alpha$ increases due to the enhanced outward diffusion of angular momentum. We find that for the canonical value $\gamma=1.5$, shocked disk solutions can exist provided $\alpha \lesssim 0.27$. This establishes for the first time that highly viscous ADAF disks can contain standing shock waves. The stability of such a shock is an open question, but Nagakura \& Yamada (2008) and Okuda et al. (2007) recently found that shocks in inviscid disks are persistent, although the shock radius may oscillate. These results at least suggest the possibility that shocks in viscous disks may be stable as well, although this needs to be investigated in future work. Since shocked solutions are expected to possess higher entropy than smooth solutions, we argue based on the second law of thermodynamics that when shock solutions are permitted dynamically, they should be expected to form (Becker \& Kazanas 2001).

Although Gu \& Lu $(2001,2004)$ obtained solutions for ADAF disks containing Rankine-Hugoniot shocks, the results developed here represent the first dynamical solutions for ADAF disks with a significant level of viscosity (i.e., $\alpha=0.1$ ) containing isothermal shocks. The Rankine-Hugoniot shocks studied by Gu \& Lu $(2001,2004)$ have conserved energy transport rates, and therefore they cannot directly produce relativistic outflows (jets). Conversely, the isothermal shocks we study here have a discontinuous energy transport rate and consequently they are able to power the observed outflows. In our analysis of the disk structure, we have assumed that the accretion rate $\dot{M}$ remains constant throughout the disk, and therefore we have neglected the effect of the outflow mass loss on the dynamics of the disk, despite the fact that an outflow emanates from the shock location in our model. This assumption is probably reasonable since estimates show that the rate of mass loss from the shock into the wind is typically $\sim 10^{-3} \dot{M}$ if the jet is hadronic (Le \& Becker 2005); this ratio is further reduced by the factor $m_{e} / m_{p}$ if the jet is leptonic. However, there are two other potential forms of mass loss from the disk that must also be considered. The first is the possibility of hydrodynamically driven outflows of the background thermal gas, and the second is the possibility of the escape of a significant number of relativistic particles from the inner and/or outer regions of the disk, rather than from the shock itself. We consider each of these issues below.

Narayan et al. (1997) and Blandford \& Begelman (1999) have noted that ADAF disks typically possess a positive Bernoulli parameter,

$$
B(r) \equiv \frac{v^{2}}{2}+\frac{\ell^{2}}{2 r^{2}}+\frac{\gamma a^{2}}{\gamma-1}-\frac{G M}{r-r_{\mathrm{s}}},
$$

suggesting that sufficient energy is available to power hydrodynamical outflows of the thermal gas. It is interesting to re-examine this issue in the context of the shocked, viscous accretion disks considered here. In Figures 10(a) and (b), respectively, we plot the Bernoulli parameter as a function of radius based on the smooth and shocked models for M87 and Sgr A* developed in Section 5. Both the smooth and shocked solutions display a slightly positive Bernoulli parameter in the inner and outer regions, suggesting that the gas is unbound there. When a shock is present, the gas is bound in the post-shock region, but it eventually becomes unbound again before crossing the horizon.

The Bernoulli parameter is related to the energy transport rate $\dot{E}$ and the viscous torque $\mathcal{G}$ via (see Equation (3))

$$
\dot{E}=-\mathcal{G} \Omega+\dot{M} B .
$$




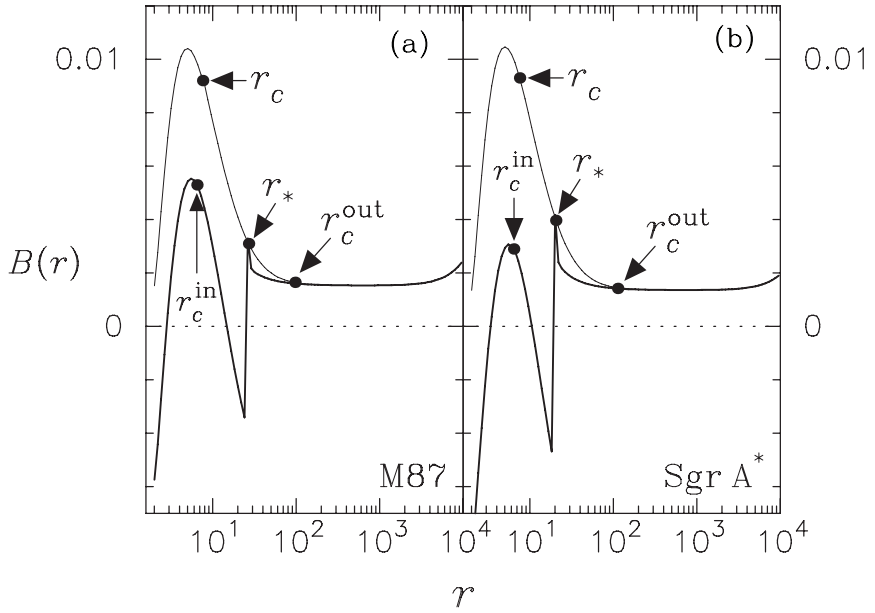

Figure 10. Bernoulli parameter (Equation (36)) plotted as a function of the radius $r$ in units of $G M / c^{2}$ for (a) M87 and (b) Sgr A*. The thick and thin lines denote the shocked and smooth solutions, respectively.

Since $\dot{E}$ is conserved in the inner region, it follows that the positive bump in the Bernoulli parameter between the shock and the horizon reflects an increase in the torque-driven outward energy transport rate, $\mathcal{G} \Omega$. This effect is less pronounced in the shocked solution because the escaping particles carry away a significant fraction of the binding energy. The positivity of the Bernoulli parameter in the inner and outer regions suggests the possibility of hydrodynamical outflows emanating from the non-shock regions of the flow. However, any such outflows would be quite weak, with terminal Lorentz factors $\Gamma=B+1 \sim 1.01$, whereas the terminal Lorentz factor for the outflow from the shock is $\Gamma \sim 6$ (Becker et al. 2008; P. A. Becker et al. 2009, in preparation). More fundamentally, it must be emphasized that despite the positivity of the Bernoulli parameter, there is no hydrodynamical mechanism in the model considered here capable of generating an outflow, since the ADAF disks we focus on are in vertical hydrostatic equilibrium, and therefore the net vertical force vanishes (cf. Equation (13)). Hence some additional mechanism, outside the standard ADAF framework, must be operative in order to channel the energy into the outflows. In our view, the outflows are not hydrodynamical in nature, but are instead powered by the first-order Fermi acceleration of relativistic particles in the vicinity of the discontinuous shock.

In addition to the possibility of hydrodynamical mass loss, some mass may also be lost from the disk in the form of relativistic particles that escape from the inner and outer regions of the disk, rather than from the shock itself. The escape of these additional relativistic particles could be driven by the distributed first-order Fermi acceleration occurring throughout the disk as a consequence of the overall compression of the flow. The significance of this effect can only be understood by performing a detailed study of the relativistic particle transport occurring throughout the disk, which is beyond the scope of this paper. We have developed such a model and will report the results in a separate paper. Preliminary calculations suggest that the spatial distribution of the escaping particles is strongly peaked at the shock location, with about half of the particles escaping from the shock itself, and the remainder escaping from the surrounding region. Hence, we conclude that the loss of mass from the disk is strongly concentrated in the vicinity of the shock, and that it is dominated by the escape of relativistic particles, rather than by hydrodynamical outflows of the background (thermal) gas.
The authors thank the anonymous referee for providing several useful comments that significantly strengthened the paper. S.D. acknowledges support via a postdoctoral fellowship from the Korea Astronomy and Space Science Institute (KASI).

\section{REFERENCES}

Abramowicz, M. A., \& Chakrabarti, S. K. 1990, ApJ, 350, 281

Abramowicz, M. A., \& Zurek, W. H. 1981, ApJ, 246, 314

Becker, P. A., Das, S., \& Le, T. 2008, ApJ, 677, L93

Becker, P. A., \& Kazanas, D. 2001, ApJ, 546, 429

Becker, P. A., \& Le, T. 2003, ApJ, 588, 408

Becker, P. A., \& Subramanian, P. 2005, ApJ, 622, 520

Bicknell, G. V., \& Begelman, M. C. 1996, ApJ, 467, 597

Blandford, R. D., \& Begelman, M. C. 1999, MNRAS, 303, L1

Caditz, D. M., \& Tsuruta, S. 1998, ApJ, 501, 242

Chakrabarti, S. K. 1989a, PASJ, 41, 1145

Chakrabarti, S. K. 1989b, ApJ, 347, 365

Chakrabarti, S. K. 1990, Theory of Transonic Astrophysical Flows (Singapore: World Scientific)

Chakrabarti, S. K. 1996, ApJ, 464, 664

Chakrabarti, S. K., Acharyya, K., \& Molteni, D. 2004, A\&A, 421, 1

Chakrabarti, S. K., \& Das, S. 2004, MNRAS, 349, 649

Chakrabarti, S. K., \& Molteni, D. 1993, ApJ, 417, 671

Das, S. 2007, MNRAS, 376, 1659

Das, S., \& Chakrabarti, S. K. 2004, Int. J. Mod. Phys. D, 13, 1955

Das, S., Chattopadhyay, I., \& Chakrabarti, S. K. 2001, ApJ, 557, 983

Das, T. K., Pendharkar, J. K., \& Mitra, S. 2003, ApJ, 592, 1078

Falcke, H., \& Biermann, P. L. 1999, A\&A, 342, 49

Ford, H. C., et al. 1994, ApJ, 435, L27

Frank, J., King, A. R., \& Raine, D. J. 2002, Accretion Power in Astrophysics (Cambridge: Cambridge Univ. Press)

Fukue, J. 1987, PASJ, 39, 309

Fukumura, K., \& Tsuruta, S. 2004, ApJ, 611, 964

Gu, W.-M., \& Lu, J.-F. 2001, Chin. Phys. Lett., 18, 148

Gu, W.-M., \& Lu, J.-F. 2004, Chin. Phys. Lett., 21, 2551

Hawley, J. F., Smarr, L. L., \& Wilson, J. R. 1984a, ApJ, 277, 296

Hawley, J. F., Smarr, L. L., \& Wilson, J. R. 1984b, ApJS, 55, 211

Kovalenko, I. G., \& Lukin, D. V. 1999, Astron. Lett., 25, 215

Le, T., \& Becker, P. A. 2004, ApJL, 617, L25

Le, T., \& Becker, P. A. 2005, ApJ, 632, 476

Le, T., \& Becker, P. A. 2007, ApJ, 661, 416

Lightman, A. P., \& Eardley, D. M. 1974, ApJ, 187, L1

Lu, J. F., \& Yuan, F. 1998, MNRAS, 295, 66

Ma, R.-Y., Yuan, F., \& Wang, D.-X. 2007, ApJ, 671, 1981

Molteni, D., Sponholz, H., \& Chakrabarti, S. K. 1996, ApJ, 457, 805

Muchotrzeb, B., \& Paczyński, B. 1982, Acta Astron., 32, 1

Nagakura, H., \& Yamada, S. 2008, ApJ, 689, 391

Narayan, R., Kato, S., \& Honma, F. 1997, ApJ, 476, 49

Narayan, R., \& McClintock, J. E. 2008, New Astron. Rev., 51, 733

Nobuta, K., \& Hanawa, T. 1994, PASJ, 46, 257

Novikov, I., \& Thorne, K. S. 1973, in Black Holes, ed. C. DeWitt \& B. DeWitt (New York: Gordon and Breach), 343

Okuda, T., Teresi, V., \& Molteni, D. 2007, MNRAS, 377, 1431

Owen, F. N., Eilek, J. A., \& Kassim, N. E. 2000, ApJ, 543, 611

Paczyński, B., \& Bisnovatyi-Kogan, G. 1981, Acta Astron., 31, 283

Paczyński, B., \& Wiita, P. J. 1980, A\&A, 88, 23

Quataert, E. 2003, Astron. Nachr., 324, 435

Reif, F. 1965, Fundamentals of Statistical and Thermal Physics (New York: McGraw-Hill)

Reynolds, C. S., et al. 1996, MNRAS, 283, L111

Ryu, D., Chakrabarti, S. K., \& Molteni, D. 1997, ApJ, 474, 378

Schödel, R., et al. 2002, Nature, 419, 694

Shakura, N. I., \& Sunyaev, R. A. 1973, A\&A, 24, 337

Takahashi, R. 2007, A\&A, 461, 393

Weinberg, S. 1972, Gravitation and Cosmology (New York: Wiley)

Yang, R., \& Kafatos, M. 1995, A\&A, 295, 238

Yu, C., Lou, Y. Q., Bian, F. Y., \& Wu, Y. 2006, MNRAS, 370, 121

Yuan, F. 2000, MNRAS, 319, 1178

Yuan, F. 2007, in ASP Conf. Ser. 373, The Central Engine of Active Galactic

Nuclei, ed. L. C. Ho \& J.-M. Wang (San Francisco, CA: ASP), 95

Yuan, F., Dong, S., \& Lu, J. F. 1996, Ap\&SS, 246, 197

Yuan, F., Ma, R., \& Narayan, R. 2008, ApJ, 679, 984

Yuan, F., Markoff, S., \& Falcke, H. 2002, A\&A, 383, 854 